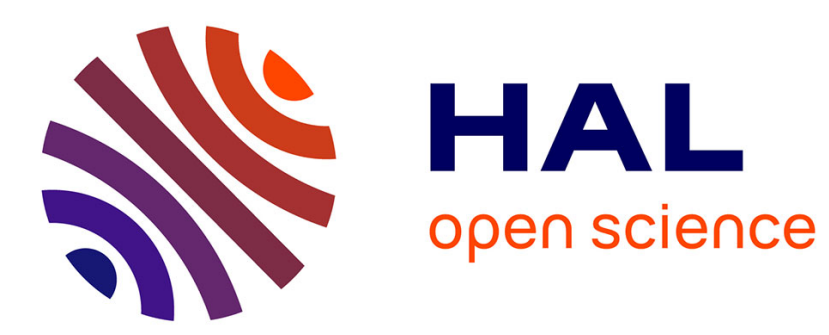

\title{
Les stabilisations des années vingt: réflexions sur la notion de crédibilité
}

\author{
Mathilde Maurel
}

\section{To cite this version:}

Mathilde Maurel. Les stabilisations des années vingt: réflexions sur la notion de crédibilité. Revue Economique, 1997, 48 (3), pp.539-548. hal-01010328

\section{HAL Id: hal-01010328 \\ https://hal-sciencespo.archives-ouvertes.fr/hal-01010328}

Submitted on 19 Jun 2014

HAL is a multi-disciplinary open access archive for the deposit and dissemination of scientific research documents, whether they are published or not. The documents may come from teaching and research institutions in France or abroad, or from public or private research centers.
L'archive ouverte pluridisciplinaire HAL, est destinée au dépôt et à la diffusion de documents scientifiques de niveau recherche, publiés ou non, émanant des établissements d'enseignement et de recherche français ou étrangers, des laboratoires publics ou privés. 


\title{
Persée
}

http://www.persee.fr

\section{Les stabilisations des années vingt. Réflexions sur la notion de crédibilité.}

\author{
Mathilde Maurel \\ Revue économique, Année 1997, Volume 48, Numéro 3 \\ p. $539-548$
}

Voir l'article en ligne

\section{Avertissement}

L'éditeur du site «PERSEE » - le Ministère de la jeunesse, de l'éducation nationale et de la recherche, Direction de l'enseignement supérieur, Sous-direction des bibliothèques et de la documentation - détient la propriété intellectuelle et les droits d'exploitation. A ce titre il est titulaire des droits d'auteur et du droit sui generis du producteur de bases de données sur ce site conformément à la loi n`98-536 du 1 er juillet 1998 relative aux bases de données.

Les oeuvres reproduites sur le site «PERSEE » sont protégées par les dispositions générales du Code de la propriété intellectuelle.

Droits et devoirs des utilisateurs

Pour un usage strictement privé, la simple reproduction du contenu de ce site est libre.

Pour un usage scientifique ou pédagogique, à des fins de recherches, d'enseignement ou de communication excluant toute exploitation commerciale, la reproduction et la communication au public du contenu de ce site sont autorisées, sous réserve que celles-ci servent d'illustration, ne soient pas substantielles et ne soient pas expressément limitées (plans ou photographies). La mention Le Ministère de la jeunesse, de l'éducation nationale et de la recherche, Direction de l'enseignement supérieur, Sous-direction des bibliothèques et de la documentation sur chaque reproduction tirée du site est obligatoire ainsi que le nom de la revue et- lorsqu'ils sont indiqués - le nom de l'auteur et la référence du document reproduit.

Toute autre reproduction ou communication au public, intégrale ou substantielle du contenu de ce site, par quelque procédé que ce soit, de l'éditeur original de l'oeuvre, de l'auteur et de ses ayants droit.

La reproduction et l'exploitation des photographies et des plans, y compris à des fins commerciales, doivent être autorisés par l'éditeur du site, Le Ministère de la jeunesse, de l'éducation nationale et de la recherche, Direction de l'enseignement supérieur, Sous-direction des bibliothèques et de la documentation (voir http://www.sup.adc.education.fr/bib/ ). La source et les crédits devront toujours être mentionnés. 


\title{
Les stabilisations des années vingt
}

\author{
Réflexions sur la notion de crédibilité
}

Mathilde Maurel*

\begin{abstract}
Les années vingt constituent un laboratoire pour l'analyse des phénomènes monétaires. Sargent [1982] insiste sur le rôle de la crédibilité dans le succès de la politique économique. Dans un modèle centré sur le financement monétaire du déficit, la thèse selon laquelle les années vingt constituent un paradigme de la politique économique est remise en question. Le modèle est validé empiriquement par les hyperinflations et stabilisations hongroise et allemande.
\end{abstract}

\section{STABILIZATIONS IN THE TWENTIES : THOUGHTS ON THE CREDIBILITY CONCEPT}

The twenties are akin to laboratory experiments which are useful for the analysis of monetary events. Sargent (1982) highlights the role of credibility in the success of economic policy and the examplarity of the twenties for understanding contemporaneous stakes. Our model is based on the monetary financing of the deficit ; hence it suggests that any generalization is cautious. Two empirical illustrations are proposed : the German and Hungarian hyperinflation and stabilization processes.

Classification JEL : E41, E62, N14

\section{INTRODUCTION}

La Hongrie a traversé deux expériences de stabilisation. La première, avec un véritable changement de régime et un dispositif de réformes fiscales crédibles, a échoué. Dans la seconde, la Société des Nations (SDN) favorisait la réinsertion de la Hongrie dans les marchés internationaux, ce qui mettait un terme au financement monétaire inéluctable du déficit, et à l'effet Oliveira-Tanzi (O-T). Dans un modèle simple, on montre que cet effet engendre des équilibres multiples, correspondant à des hyperinflations distinctes. L'analyse empirique des inflations allemande et hongroise suggère que chacune renvoie à un type différent, et que le modèle décrit correctement les processus inflationnistes des années vingt.

\footnotetext{
* CNRS (ROSES)/OFCE/CEPR, 69 Quai d'Orsay, 75340 Paris Cedex 07.
} 
Les implications de cette analyse formelle sont nombreuses ${ }^{1}$. Sargent insiste sur le rôle de la crédibilité des réformes fiscales et sur le changement de régime qu'elles impliquent. La normalisation des relations internationales avec la Hongrie, étape majeure au lendemain de la première guerre mondiale, a sans aucun doute crédibilisé la politique hongroise. Toutefois la crédibilité est une notion complexe qui ne se réduit pas à un événement de politique internationale précis. Selon Sargent, les hyperinflations des années vingt, sorte de cas limites, constituent un paradigme qui permet de trancher le dilemme de la politique économique dans les années quatre-vingt. Or, si les années vingt sont caractérisées par le développement d'un effet $\mathrm{O}-\mathrm{T}$, elles ne peuvent pas être généralisées à une période dans laquelle le recours au financement monétaire du déficit n'existe pas.

\section{LES DEUX STABILISATIONS HONGROISES}

\section{Première tentative de stabilisation}

En 1921, le Docteur R. Hegedüs fut investi de pouvoirs quasi dictatoriaux pendant un an et tenta de mettre en place une politique de stabilisation financière. Cette politique comportait trois volets : l'acceptation des réparations; la mise en place d'une fiscalité qui devait permettre de réaliser l'équilibre des dépenses et des recettes; enfin la création d'un institut d'émission qui n'était pas indépendant du gouvernement, mais dont le pouvoir d'émission était limité à 22 milliards de couronnes.

L'échec de cette tentative, qui pourrait correspondre au seul véritable changement de régime dans les années vingt en Hongrie $^{2}$, suggère que l'annonce et la mise en place d'une politique fiscale cohérente étaient insuffisantes pour stopper l'inflation. Il fallait que l'arrêt de l'inflation précédât la réforme afin de restaurer mécaniquement le niveau des recettes.

L'effet O-T décrit l'impact négatif de l'inflation sur les revenus fiscaux indexés sur l'année précédente. Or l'inflation était alimentée par le traité de Trianon luimême, qui, en restreignant l'accès aux capitaux étrangers pour financer le déficit, rendait plus inéluctable son financement monétaire : «One of the objects pursued by Dr. Hegedüs in undertaking drastic measures for the balancing of the budget, was to create a condition, in which Hungary might be able to raise foreign loans for the purpose of making up the deficit in her balance of payments. Such loans were unthinkable, however, as long as the reparation problem remained in the indefinite situation in which the treaty of Trianon had left it ${ }^{3}$. » L'adoption d'une discipline fiscale destinée à rassurer d'éventuels prêteurs internationaux avait des

1. Notamment, l'idée selon laquelle l'hyperinflation ne correspond pas à l'existence de bulles est remise en question (voir Flood et Garber [1980], Hamilton et Whiteman [1985], Casella [1989], P. Michael, A.R. Nobay et D.A. Peel [1994].

2. "The present study argues that the 1921 reforms, previously largely ignored in the literature, produced a change in the monetary regime. By contrast, the post-1924 regime, previously assumed to be a distinct period (Sargent, [1986], chap. 4) turns out to be largely indistinguishable from the one which preceded it."(Pierre L. Syklos, p. 274).

3. Pasvolsky [1928], p. 307. 
effets limités tant que persistait l'hypothèque sur les biens de la Hongrie et la prédominance des réparations sur tous les autres emprunts.

\section{Deuxième stabilisation réussie grâce à l'intervention de la SDN}

La deuxième politique de stabilisation a reposé essentiellement sur la réinsertion de la Hongrie dans l'économie mondiale. Le 21 février 1924, la Commission des réparations acceptait de lever l'hypothèque qui pesait sur les ressources hongroises. Un prêt de 250 millions de couronnes put donc être négocié et fut employé à stabiliser la couronne hongroise.

La disparition de l'effet $\mathrm{O}-\mathrm{T}$ et la réussite de la stabilisation sont-elles dues à la seule crédibilité de la politique monétaire et fiscale ? À condition de priver la définition qu'en donne Sargent d'une grande partie de sa substance. Si la structure des dépenses est modifiée (licenciements administratifs, réduction du coût des chemins de fer), leur niveau reste invariant (augmentation du salaire réel des fonctionnaires, paiement d'indemnités aux chômeurs). Du côté des recettes, la contribution de nouveaux impôts est seulement marginale : l'introduction d'une taxe foncière en Hongrie représente seulement $3,7 \%$ des recettes totales. Le tableau 1 montre l'amélioration mois par mois du taux de couverture des dépenses et suggère que l'arrêt de l'inflation, plus que les réformes structurelles dont les délais de mise en place excèdent le mois', est à l'origine du succès de la stabilisation. Selon la Société des Nations ${ }^{2}$ : «The required increase in revenue may be expected from the automatically better returns (in terms of gold value) from existing taxes (as in the case of Austria) when the stabilisation of the currency is effected »; « budget equilibrium followed [the stabilisation], it did not precede $^{3}$. »

\section{UN MODĖLE POUR L'HYPERINFLATION ET SON ILLUSTRATION EMPIRIQUE}

Dans un article de 1990, Franco suggère que l'existence d'un effet O-T relègue au second plan le rôle de la crédibilité des réformes fiscales soulignée par Sargent [1982] (on a vu dans la section précédente que le double cas de la Hongrie corroborait cette hypothèse). Le modèle qui suit met en évidence des équilibres multiples qui sont dus à cet effet. Deux configurations d'hyperinflation y sont possibles. Dans la première, si le ratio du déficit financé par création monétaire sur l'inflation est croissant, et supérieur à un seuil critique, les anticipations et les variables fondamentales de l'économie (le PIB et les encaisses réelles) ne sont plus coïntégrées. Dans la seconde, le ratio du déficit financé par création

1. Selon Pasvolsky [1928, p. 306], les délais de mise en place des réformes fiscales sont très longs: «White it was easy enough to obtain the passage by the National Assembly of the twenty-nine financial bills submitted by him during his regime, it was very much more difficult to organize the administrative machinery for the execution of the measures introduced. The result of the drastic increases of taxes were much too slow to bear adequately upon the budgetary situation $»$.

2. Société des Nations [1926], p. 64-66.

3. Société des Nations [1926], p. 37. 
Tableau 1. Budgets mensuels hongrois.

\begin{tabular}{l|c|c|r|c}
\hline & Dépenses & & & $\begin{array}{c}\text { Recettes en pourcentages } \\
\text { des dépenses }\end{array}$ \\
\hline 1 & 30.5 & 17.9 & -12.6 & 58.7 \\
2 & 33.8 & 29.5 & -4.3 & 87.3 \\
3 & 36.7 & 30.9 & -5.8 & 84.2 \\
4 & 33.7 & 35.6 & 1.9 & 105.6 \\
5 & 34.0 & 49.2 & 15.2 & 144.7 \\
6 & 37.2 & 44.9 & 7.7 & 120.7 \\
\hline Total & 205.9 & 208.0 & 2.1 & 101.0 \\
\hline
\end{tabular}

Franco [1990], p. 178 : Les données proviennent des Rapports mensuels du Commissaire général de la Société des Nations. Pour l'Autriche, elles sont déflatées avec les taux de change de Walré de Bordes [1924, p. 114-139]. Pour la Hongrie, les données proviennent aussi de Young [1925, vol. II, p. 326].

monétaire sur l'inflation est décroissant, l'inflation est plus élevée que dans le cas précédent, et on retrouve une relation de coïntégration entre les encaisses réelles et l'inflation '. L'illustration du modèle par les hyperinflations allemande et hongroise des années vingt renforce de manière plus formelle l'argument de Franco.

La demande d'encaisses monétaires $\mathrm{x}$ dépend négativement du taux d'intérêt nominal $i$ et positivement du volume de transactions $y$ dans l'économie :

$$
i=k y-f x
$$

Le niveau de la production y dépend de la compétitivité de l'économie, c'està-dire du taux de change réel $e-p$ :

$$
y=a(e-p)
$$

L'écart de la production $y$ par rapport au revenu «normal » $\bar{y}$ détermine le niveau de l'inflation $\dot{p}$ (courbe de Phillips) :

$$
\dot{p}=\alpha(y-\bar{y})
$$

Si l'accroissement des encaisses nominales $\frac{\dot{M}}{\mathrm{P}}$ sert à financer le déficit budgétaire $\mathrm{D}(\dot{p})$, avec $\mathrm{D}^{\prime}(\dot{p})>0^{2}: \frac{\mathrm{M}}{\mathrm{P}}=\mathrm{D}(\dot{p})$

On peut écrire :

$$
\begin{gathered}
\left(\frac{\dot{\mathrm{M}}}{\mathrm{P}}\right) /\left(\frac{\mathrm{M}}{\mathrm{P}}\right)=\frac{\mathrm{D}(\dot{p})}{(\mathrm{M} / \mathrm{P})}-\dot{p} \\
\Leftrightarrow \dot{x}=\mathrm{D}(\dot{p}) e^{-x}-\dot{p}
\end{gathered}
$$

$\operatorname{avec} x=\log \left(\frac{M}{P}\right)$.

1. Ce type de configuration est étudié par P. Michael, A.R. Nobay et D.A. Peel [1994].

2. Le déficit augmente avec l'inflation. 
L'équation (4) dit que le taux de croissance de la masse monétaire réelle est égal au taux de croissance de la masse monétaire nominale moins une taxe inflationniste.

Enfin, la condition d'arbitrage sur le marché des capitaux s'écrit :

$$
i=\dot{e}+i^{*}
$$

où $i^{*}$ est le taux d'intérêt mondial.

La forme réduite et linéarisée autour des équilibres donne deux équations $\dot{e}-\dot{p}=0$ et $\stackrel{x}{x}=0$, qui peuvent être représentées dans le plan (E, $x$ ) suivant. On suppose que $k-\alpha<0$ est négatif, ce qui implique que le long de la droite $\dot{e}-\dot{p}=0$, le niveau des encaisses réelles dépend négativement de l'inflation, comme dans le modèle de Cagan [1956].

\section{Diagramme de phase}

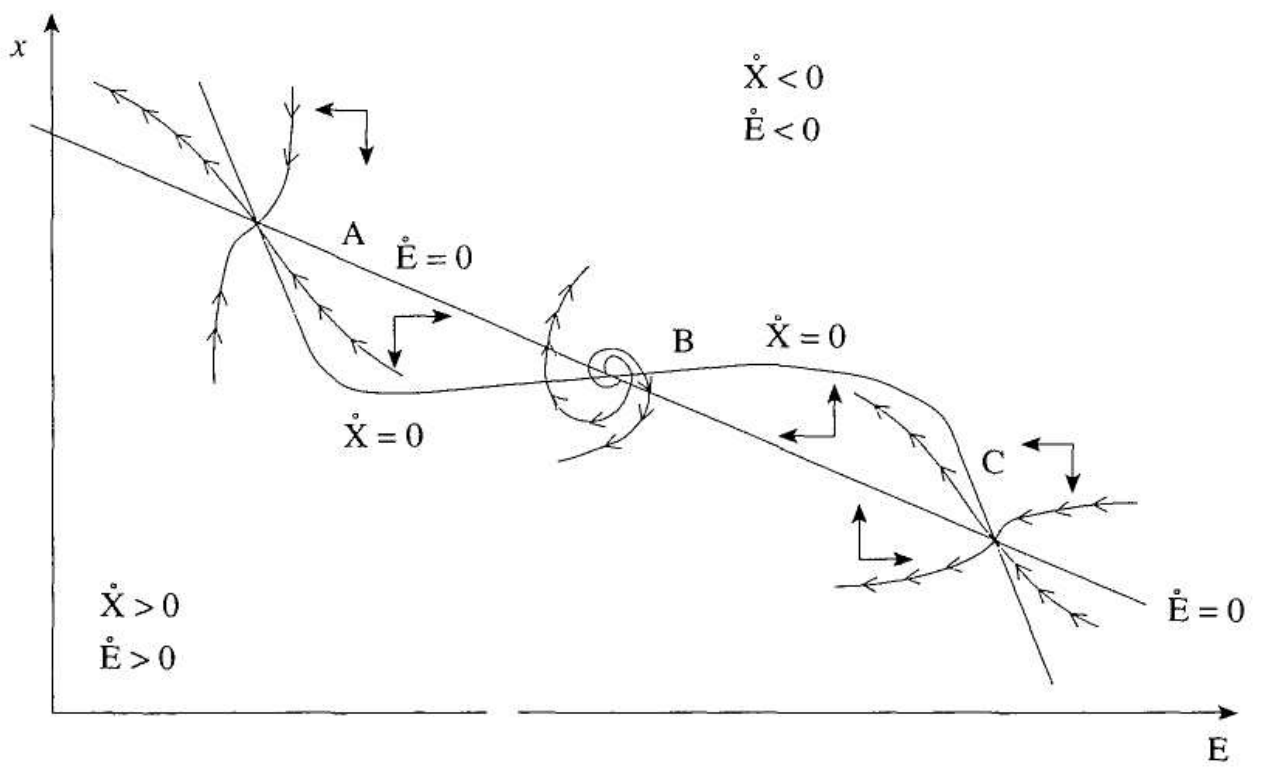

$$
\left.\frac{d x}{d \mathrm{E}}\right|_{\substack{\begin{subarray}{c}{x=0 \\
\mathrm{E}=\mathrm{E}_{0}} }} \\
{ }\end{subarray}}=\mathrm{P} \text {, pente de la fonction } \stackrel{\circ}{x}=0 \Leftrightarrow e^{x}=\frac{\mathrm{D}[\dot{p}]}{\dot{p}} \text { autour des }
$$
$\mathrm{E}=\mathrm{E}_{0}$
équilibres, mesure l'intensité de l'effet Tanzi : $\frac{\mathrm{D}[\dot{p}]}{\dot{p}}$ est décroissant lorsque l'inflation est soit très forte (la croissance de la masse monétaire ne peut plus rattraper l'inflation), soit faible (l'inflation croît plus vite que la partie du déficit qui est monétisée) ; le ratio est croissant dans la région intermédiaire : les revenus fiscaux, indexés sur l'année précédente, diminuent, et le déficit monétisé augmente plus vite que l'inflation.

L'étude du modèle donne les conditions de stabilité suivantes. 


\section{Cas 1}

Si P est inférieur à $\frac{k-\alpha}{f \alpha}$, le discriminant est positif et les racines, réelles, sont de signes opposées. Les équilibres sont alors des points selles ${ }^{1}$.

\section{Cas 2}

Si P est compris entre $\frac{k-\alpha}{f \alpha}$ et $\frac{\left(a(k-\alpha)+\dot{p}_{0}\right)^{2}}{4 f a \alpha \dot{p}_{0}}>\frac{k-\alpha}{f \alpha}$, les trajectoires sont nodales. Elles sont convergentes lorsque $y_{0}<\frac{a(k-\alpha)}{\alpha}+\bar{y}<\bar{y}$, c'est-à-dire lorsque le niveau du revenu en 0 , qui n'est pas le revenu de long terme, et qui est financé de manière inflationniste (équation 3), est suffisamment proche du revenu de long terme $\bar{y}$.

\section{Cas 3}

Si P est supérieur à $\frac{\left(a(k-\alpha)+\dot{p}_{0}\right)^{2}}{4 f a \alpha \dot{p}_{0} a(k-\alpha)}$, les trajectoires sont sinusoïdales, et convergentes à nouveau lorsque $y_{0}<\frac{a(k-\alpha)}{\alpha}+\bar{y}<\bar{y}$.

Le modèle permet de distinguer deux types d'hyperinflation.

\section{Premier type d'hyperinflation}

Dans les années vingt, l'importance des déficits budgétaires financés par émission monétaire suggère que l'économie se trouve au voisinage des points $\mathrm{B}$ ou $\mathrm{C}$.

Dans le voisinage du point $\mathrm{B}$, si $\mathrm{P}$ est tel que la trajectoire de l'économie est sinusoïdale, l'économie peut «sauter » à droite ou à gauche de $\mathrm{B}$. Cette situation traduit le fait que le lien entre l'inflation et la demande d'encaisses réelles n'existe plus. Les fondamentaux économiques ne déterminent plus le rythme de l'inflation et si le point B n'est pas stable, l'inflation devient explosive. P est déterminée par les conditions initiales : ampleur du déficit financé de manière inflationniste, gravité des déséquilibres engendrés par la reconstruction, rationnement des crédits sur les marchés internationaux. Une politique de stabilisation avisée consiste à mettre en œuvre des moyens pour résorber ces déséquilibres ${ }^{2}$ et pour diminuer le recours au financement monétaire du déficit. Une telle politique est de manière tautologique crédible. Mais la seule crédibilité des réformes n'est pas suffisante ${ }^{3}$.

\section{Deuxième type d'hyperinflation}

Il existe en effet au moins un autre type d'hyperinflation. P. Michael, A.R. Nobay et D.A. Peel [1994] montrent que, dans le contexte allemand des années vingt, les variables d'encaisses réelles et d'inflations sont coïntégrées ${ }^{4}$. Ce

1. B ne peut pas être un point selle (au voisignage de $\mathrm{B}, \mathrm{P}$ est positif).

2. En rendant accessibles les marchés de capitaux internationaux par exemple.

3. Voir à cet égard l'échec du premier épisode de stabilisation hongroise.

4. À condition de bien spécifier le modèle, en ajoutant un proxy pour les fluctuations du PIB qui est le salaire nominal, et en distinguant la période d'hperinflation des mois qui la précèdent. 
régime d'hyperinflation correspond au point $\mathrm{C}$, qui est un point selle, autour duquel il existe deux trajectoires linéaires et convergentes.

Si l'Allemagne se trouve, à la différence de la Hongrie, dans la région $\mathrm{C}$ du diagramme précédent, l'inflation est telle que le rapport du déficit monétisé sur l'inflation est décroissant. C'est bien ce que nous trouvons dans la dernière colonne du tableau 2.

Tableau 2. Allemagne. Recettes et dépenses (mai 1923-décembre 1923)

(millions de marks or)

\begin{tabular}{l|r|r|r|r|r}
\hline \multicolumn{1}{c|}{ Période } & Dépenses & Recettes & $\begin{array}{r}\text { Recettes } \\
\text { en pourcentages } \\
\text { des dépenses }\end{array}$ & Inflation & $\begin{array}{c}\text { Rapport } \\
\text { du déficit } \\
\text { sur l'inflation }\end{array}$ \\
\hline 1923 & 284.7 & 123.3 & 43.3 & 1.93 & 83.69 \\
Mai & 496.4 & 48.2 & 9.7 & 3.03 & 148.14 \\
Juin & 473.9 & 48.3 & 10.2 & 6.98 & 60.99 \\
Juillet & 883.6 & 11.7 & 1.3 & 17.89 & 48.72 \\
Août & 689.2 & 14.4 & 2.1 & 86.70 & 7.78 \\
Septembre & 235.8 & 1.2 & 0.5 & 174.08 & 1.35 \\
Octobre & 134.2 & 0.1 & 0.1 & 13.33 & 10.06 \\
Novembre 1-10 & 28.5 & 0.4 & 1.5 & & \\
$10-20$ & 258.7 & 10.6 & 4.1 & & \\
$20-30$ & 421.4 & 11.1 & 2.6 & & \\
\hline Total & 179.9 & 32.8 & 18.2 & & \\
\hline Décembre 1-10 & 165.7 & 42.9 & 25.9 & & \\
10-20 & 153.8 & 88.8 & 57.8 & & \\
20-31 & 499.4 & 164.5 & 32.9 & & \\
\hline Total & & & & & \\
\hline
\end{tabular}

Source : trois premières colonnes : Franco [1990, p. 183] ; quatrième colonne (inflation) : Young [1925]; cinquième colonne : nos calculs.

Dans le cas hongrois, le problème est étudié sous un angle différent. Ne disposant pas de statistiques mensuelles sur le déficit, on étudie l'évolution de l'inflation et des encaisses réelles. Si l'on se trouve effectivement dans la région $\mathrm{B}$, si le ratio du déficit hongrois sur l'inflation est croissant, et que l'effet Tanzi est suffisamment fort, la trajectoire de l'économie devient sinusoïdale. Statistiquement, cela se traduit par le développement d'une bulle.

La relation de $\operatorname{Cagan}^{1}$ que nous estimons porte sur la période allant de juillet 1921 à juillet 1924, en Hongrie :

$$
x_{t}=\delta-\alpha \pi_{t}+\beta y_{t}+v_{t}
$$

1. La forme linéarisée et réduite du modèle précédent comporte une relation qui exprime la demande d'encaisses réelles en fonction du taux de change. La combinaison des équations (2) et (3) permet de retrouver une fonction similaire à (5). 
$x_{t}:$ logarithme des encaisses réelles, données extraites de Young [1925] ;

$\pi_{t}$ : logarithme de l'inflation anticipée ;

$y_{t}:$ logarithme du PIB ;

La demande d'encaisses réelles dépend de l'inflation anticipée et des fluctuations de la production.

Ne disposant, pour la Hongrie, ni de l'inflation anticipée, ni de la production mensuelle, on fait les deux hypothèses suivantes :

$$
\begin{gathered}
\eta_{1 t}=\text { trend }-y_{t} \rightarrow \mathrm{I}(0) \\
\eta_{2 t}=\Delta p_{t}-\pi_{t} \rightarrow \mathrm{I}(0)
\end{gathered}
$$

$\Delta p_{t}=p_{t}-p_{t}$, où $p_{t}$ est un indice de prix mensuel (données extraites de Young [1925]).

Si dans le modèle (5), les variables sont coïntégrées, et si le résidu est stationnaire, alors on peut remplacer le PIB par le trend ${ }^{1}$ et l'inflation anticipée par l'inflation réalisée, et dans le modèle suivant:

$$
x_{t}=\delta-\alpha \Delta p_{t}+\beta \text { trend }+w_{t}
$$

les variables sont coïntégrées et le résidu est stationnaire.

L'hypothèse (7) dit que l'inflation anticipée et l'inflation réalisée sont coïntégrées. Elle permet de n'exclure a priori aucune spécification pour les anticipations, qui peuvent être aussi bien adaptatives que rationnelles.

A l'instar de P. Michael, A.R. Nobay et D.A. Peel [1994], nous supposons que la non-coïntégration peut être due à une mauvaise spécification du modèle, engendrée par l'hétérogénéité de la période étudiée. Nous avons donc procédé à un test de Quandt [1972], qui détermine comme date de rupture février 1923, date de la fin de l'expérience de stabilisation de Hegedüs.

Les deux tests de coïntégration donnent les résultats suivants :

PREMIÈRE ÉTAPE. Détermination de l'ordre de stationnarité des variables à l'aide du test de Dickey-Fuller augmenté.

\begin{tabular}{l|l|l|l}
\hline \multicolumn{1}{c|}{ Période } & \multicolumn{1}{c|}{$\mathrm{x}$} & \multicolumn{1}{c|}{$\Delta p_{t}$} & \multicolumn{1}{c}{ Trend } \\
\hline De juillet 1921 à février 1923 & $\mathrm{I}(1)$ & $\mathrm{I}(1)$ & $\mathrm{I}(1)$ \\
De mars 1923 à juillet 1924 & $\mathrm{I}(1)$ & $\mathrm{I}(0)+\mathrm{C}$ & $\mathrm{I}(1)$ \\
\hline
\end{tabular}

DEUXIÈME ÉTAPE. Test de coïntégration fondé sur l'équation par les MCO suivante (voir Engle et Granger [1987]) :

$$
\Delta \hat{w}_{t}=\lambda \hat{w}_{t-1}+\varepsilon_{t} \text {, où } \hat{w}_{t} \text { est l'estimateur des résidus de l'équation (5'). }
$$

1. Nous avons testé l'hypothèse (6) à partir des données allemandes publiées par P. Michael, A.R. Nobay et D.A. Peel [1994], p. 20. Le trend et la production sont bien coïntégrés. 
Si $\lambda$ est significativement nul, les résidus sont non stationnaires, et les variables ne sont pas coïntégrées. L'hypothèse alternative $\mathrm{H}_{1}: \lambda<0$ signifie que les variables sont coïntégrées. Les seuils critiques auxquels doivent être comparées les statistiques de Student calculées dépendent du nombre de variables coïntégrées, du nombre d'observations, et du niveau de significativité désiré.

\begin{tabular}{c|c|c|c|c|c|c|c|c}
\hline Période & $\hat{\delta}$ & $\hat{\alpha}$ & $\hat{\beta}$ & $\mathrm{SE}$ & $\mathrm{R}^{2}$ & $\mathrm{DW}$ & $\hat{\lambda}$ & $t(\hat{\lambda})$ \\
\hline De juillet 1921 à février 1923 & 4.22 & -0.59 & -0.014 & 0.035 & 0.88 & 2.33 & -1.21 & -5.10 \\
\hline De mars 1923 à juillet 1924 & 3.99 & -0.85 & -0.008 & 0.061 & 0.55 & 1.03 & -0.49 & -2.00 \\
\hline
\end{tabular}

Le seuil critique est $-4.12(1 \%),-3.29(5 \%)$, et $-2.90(10 \%)^{1}$. Si dans la première période d'inflation hongroise, les variables fondamentales sont coïntégrées, elles ne le sont plus dans la deuxième période caractérisée par le déclenchement d'une hyperinflation et l'intervention de la SDN. Les tests statistiques suggèrent donc qu'à la différence du cas allemand étudié par P. Michael, A.R. Nobay et D.A. Peel [1994], la Hongrie se trouve dans une configuration où l'hyperinflation est caractérisée par le développement d'une bulle.

\section{CONCLUSION}

Le modèle développé dans cet article permet d'analyser l'hyperinflation dans un contexte d'équilibres multiples. Deux de ces équilibres correspondent à des situations d'hyperinflation. La première est caractérisée par le développement d'une bulle; c'est le cas hongrois de la deuxième période de stabilisation. La seconde correspond à une inflation beaucoup plus élevée et au rétablissement d'une relation de coïntégration entre les variables réelles et monétaires. La comparaison de deux épisodes contemporains (allemand, hongrois) permet d'illustrer la diversité des situations d'hyperinflation.

Les circonstances particulières qui entourent les épisodes de stabilisation en Europe centrale et orientale (financement monétaire du déficit, hypothèque mise sur les ressources des pays vaincus) mettent en garde contre la tentation de tirer des conclusions de politique économique pour la période contemporaine. Si les années vingt sont frappées par le développement d'un effet O-T, elles ne peuvent pas être généralisées à une période dans laquelle le recours au financement monétaire du déficit n'existe pas.

1. La source pour ces seuils est Engle et Yao [1987]. 


\section{RÉFÉRENCES BIBLIOGRAPHIQUES}

CAGAN P. [1956], «The Monetary Dynamics of Hyperinflation», dans M. FriedMAN (ed.), Studies in the Quantity of Money, Chicago, University of Chicago Press.

CAsella A. [1989], «Testing for Rational Bubbles with Exogenous or Endogenous Fundamentals », Journal of Monetary Economics, 24, p. 109-122.

ENGLE R.F., GRANGER C. W. J. [1987], « Cointegration and Error Correction : Representation, Estimation and Testing », Econometrica, 55, p. 251-276.

ENGLE R.F., Yoo [1987], « Forecasting and Testing in Cointegrated Systems », Journal of Econometrics, 35, p. 143-159.

Flood R.P., Garber P. [1980], « Market Fundamentals versus Price-Level Bubbles : The First Tests », Journal of Political Economy, 88, p. 24-58.

FRANCO G.H.B. [1990], "Fiscal Reforms and Stabilisation: Four Hyperinflation Cases », The Economic Journal, 100 (399), p. 176-188.

Hamilton J.D., Whiteman C.H. [1985], «The Observable Implications of Self-fulfilling Expectations », Journal of Monetary Economics, 16, p. 353-373.

Michael P., Nobay A.R. et Peel D.A. [1994], «The German Hyperinflation and the Demand for Money revisited ", International Economic Review, 35 (1), p. 1-22.

Pasvolsky L. [1928], Economic Nationalism of the Danubian States. New York, Macmillan.

SARGent T.J [1982], «The End of Four Big Inflations », dans RoberT E. Hall (ed.), Inflation: Cause and Effects, Chicago, p. 41-97.

Société des Nations [1926], «La reconstruction financière de la Hongrie. Rapport et principaux documents », Genève, Société des Nations.

SYKLOS P. L. [1990], « Interpreting a Change in Monetary Policy Regims : a Reappraisal of the First Hungarian Hyperinflation and Stabilization, 1921-1928 », p. 274-311.

YounG J.P. [1925], European Currency and Finance, Washington, Commission of Gold and Silver, United States Senate. 